\title{
Smart context-aware QoS-based admission control for biomedical wireless sensor networks
}

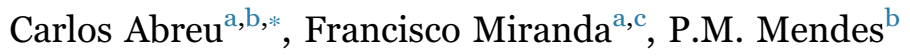 \\ a Instituto Politécnico de Viana do Castelo, Portugal \\ b CMEMS-UMinho, Universidade do Minho, Portugal \\ ${ }^{\mathrm{c}}$ Center for Research and Development in Mathematics and Applications, University of Aveiro, Portugal
}

\section{A R T I C L E I N F O}

Keywords:

Context-Aware QoS

Admission Control

Biomedical Wireless Sensor Networks

\begin{abstract}
A B S T R A C T
Wireless sensor networks are being used as the enabling technology that helps to support the development of new applications and services targeting the domain of healthcare, in particular, regarding data collection for continuous health monitoring of patients or to help physicians in their diagnosis and further treatment assessment. Therefore, due to the critical nature of both medical data and medical applications, such networks have to satisfy demanding quality of service requirements. Despite the efforts made in the last few years to develop quality of service mechanisms targeting wireless sensor networks and its wide range of applications, the network deployment scenario can severely restrict the network's ability to provide the required performance. Furthermore, the impact of such environments on the network performance is hard to predict and manage due to its random nature. In this way, network planning and management, in complex environments like general or step-down hospital units, is a problem still looking for a solution. In such context, this paper presents a smart context-aware quality of service based admission control method to help engineers, network administrators, and healthcare professionals managing and supervising the admission of new patients to biomedical wireless sensor networks. The proposed method was tested in a small sized hospital. In view of the results achieved during the experiments, the proposed admission control method demonstrated its ability, not only to control the admission of new patients to the biomedical wireless sensor network, but also to find the best location to admit the new patients within the network. By placing the new sensor nodes on the most favourable locations, this method is able to select the network topology in view of mitigating the quality of service provided by the network.
\end{abstract}

\section{Introduction}

Data gathering of physiological signals for health monitoring should not interfere with the patients' mobility. This may be achieved using wireless technology through the use of Biomedical Wireless Sensor Networks (BWSNs). Since they must be worn unobtrusively by patients, BWSNs are composed of small-sized wireless nodes equipped with sensors for biomedical data collection, designed for medical applications or healthcare services. Typical applications of BWSNs include emergency response, ambient assisted living applications to monitor and assist disabled or elderly people (Hadjidj et al., 2013; Carmen Domingo, 2012), and patient monitoring systems for chronically ill persons (Lai et al., 2009). Among these application fields, this work will focus on the aspects related to the Quality of Service (QoS) guarantees requested to BWSNs by patient monitoring systems used to collect vital and physiological signs of patients in step-down hospital units or nursing homes.

Typical patient monitoring applications, supported by BWSNs, are used to collect vital or physiological signs, such as respiratory rate, pulse rate, temperature, blood pressure, and oximetry in order to complement the measurements performed manually by nursing professionals a few times a day and thus, enhancing the quality of the health care provided to patients. The sensed data are then sent to a local or remote database to be used to support healthcare professionals on their medical practice. As an abstraction, BWSNs can be seen as the lowest layer for Healthcare Information System (HIS), collecting data to support healthcare professionals on their decisions and medical diagnosis, as shown in Fig. 1.

The use of BWSNs in healthcare can enhance the services provided to citizens. In particular, they have the potential to play an important role in the development of new real-time patient monitoring applications. However, due to the critical nature of the data carried by them, they have

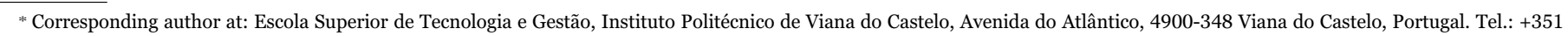
258819 700; fax: +351258 827636 .

E-mail address: cabreu@estg.ipvc.pt (C. Abreu). 


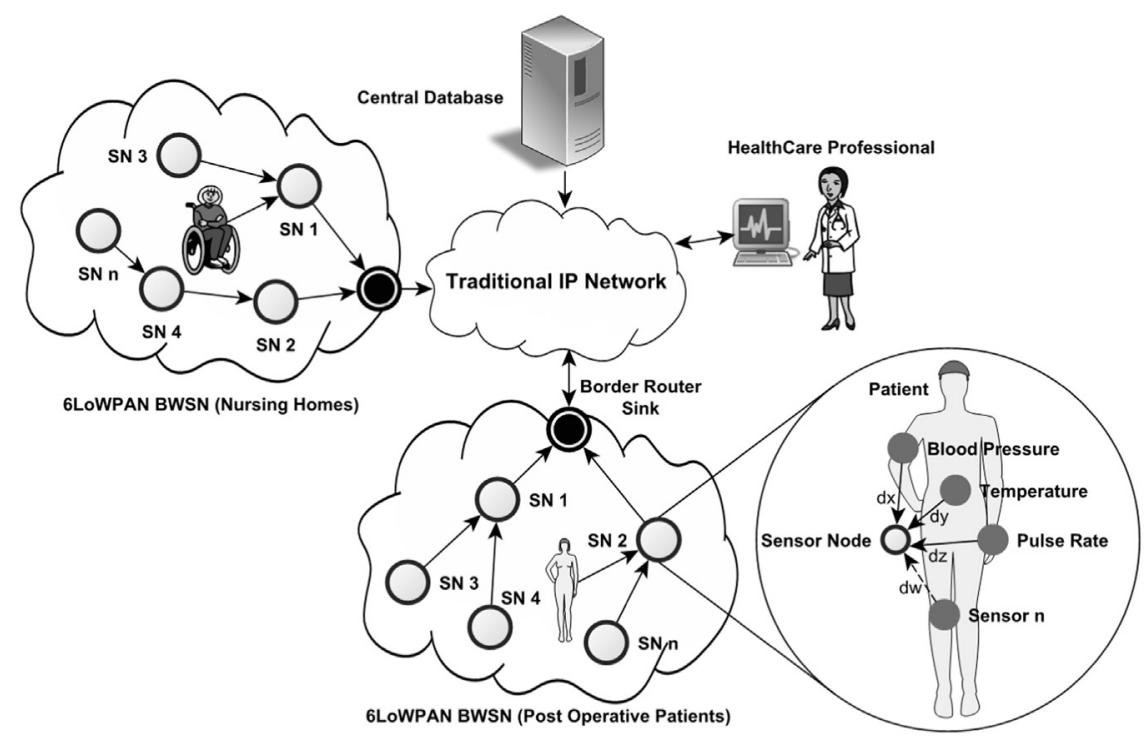

Fig. 1. Reference network architecture used to develop and test the proposed QoS probing methodology.

to fulfil high levels of QoS in order to be fully accepted by both the healthcare providers and the patients. The QoS level requested to a BWSN depend on its use, i.e., on the requirements of the application using the BWSN as communication infrastructure. Regarding the QoS requirements imposed to BWSNs (e.g., considering the signals being monitored), they can include timeliness, reliability, robustness, privacy and security (Abreu et al., 2014). On its turn, depending on the purpose of the BWSN application, QoS requirements such as mobility support or network lifetime can be very important (Abreu et al., 2014a).

The deployment of real-time monitoring applications, such as patient monitoring, is dependent on a robust communication infrastructure able to provide admission control in order to keep the required QoS levels. Despite the several QoS mechanisms proposed in the last few years, targeting Wireless Sensor Networks (WSNs) and their applications, the network deployment scenario can severely restrict the network's ability to provide the required QoS. Bearing in mind BWSNs and their typical deployment scenarios (i.e., hospitals or nursing homes), several obstacles have to be faced by engineers and network administrators. Harsh environments, like hospital facilities, can expose BWSNs to very hostile situations regarding the radio communications and thus, the network's ability to provide the required QoS. In such harsh environments the network performance depends on either random or deterministic factors. Random factors, such as the dynamics of the hospital environment, the radio interferences, and the patient's mobility can affect the network capability to provide the necessary QoS in an unexpected way (Ko et al., 2010a, 2010b). On its turn, deterministic factors, such as network congestion due to the over the populated network can be avoided using proper admission control methods.

Since the network conditions will change with time, the solution to maintain the QoS levels is to deploy algorithms to control different network aspects in order to get the best QoS (Chen et al., 2011). As a few examples, it can be mentioned the design of medium access mechanisms (Gama, 2011; Mir Mehedi et al., 2015; Anjum et al., 2013), the use of algorithms that are energy friendly (Jacob and Jacob, 2015; Khan et al., 2014), or even more complex solutions to detect and remove bad data from the network to improve the QoS (Paola et al., 2013).

Despite the many proposed mechanisms that may be used to maximise the QoS available in a WSN, in some scenarios the required levels of QoS may not be available in the network. To prevent new nodes in the network operating with QoS levels below the required by the application, the QoS level in the network must be monitored and, eventually, the admission to the network must be controlled. As discussed in Abreu et al. (2014), Pereira et al. (2012), Lindh and Orhan (2009) and Farooq and Kunz (2014), both
QoS monitoring systems and admission control systems are keystones to achieve high levels of reliability and performance in WSNs; in fact, they complement each other.

Regarding the admission control, a few approaches have been proposed in recent years. The authors of Sun et al. (2005) propose SenProbe, an end-to-end capacity estimation tool that can be used for performance analysis and network deployment planning. SenProbe uses one-way active measurements to estimate the end-to-end path capacity by injecting packet trains in the network to measure the packet dispersion. As an alternative to real-time active measurements, the authors of Yaling Yang and Kravets (2005) propose a contention-aware admission control protocol designed for IEEE 802.11 ad-hoc networks, which estimates the available channel capacity by allowing each node to estimate the time that the communication channel is being used. The work presented in Ibrahim Orhan and Lindh (2011) proposed a measurement-based admission control system, where the admission decision is made by the network coordinator, based on real-time measurements of the packet loss ratio provided by a performance meter. The performance meter runs on each sensor node and it is continually tracking the number of sent and received data packets and bytes. By running on each sensor node, the performance meter contributes to its energy depletion, reducing the network lifetime. Since WSNs are composed, typically, of energy-constrained sensor nodes, the increase of energy consumption on their nodes must be avoided. In fact, depending on the network application, it could be a strong drawback. Although proposing different solutions, all these studies support the importance and the need of using both QoS monitoring and admission control systems, to manage and improve the performance of WSNs.

In this context, the objective of this work is to contribute with a smart context-aware QoS-based admission control method for BWSNs. The proposed admission control method uses a probe based procedure to find the best location to place new patients in a nursing room or step-down hospital unit, minimising their impact on the performance of the BWSN, in order to preserve the QoS being provided to the patients already within the network. To avoid the problem of energy depletion in the energy-constrained sensor nodes, the proposed method uses the node that, usually, is plugged to the power line (i.e., the WSN border router) to collect the dataset used by the mathematical decision algorithm. To mitigate the power consumption in each sensor node, the QoS Probe was designed to be triggered for short time periods, and only on demand, leading to an insignificant extra power use. 

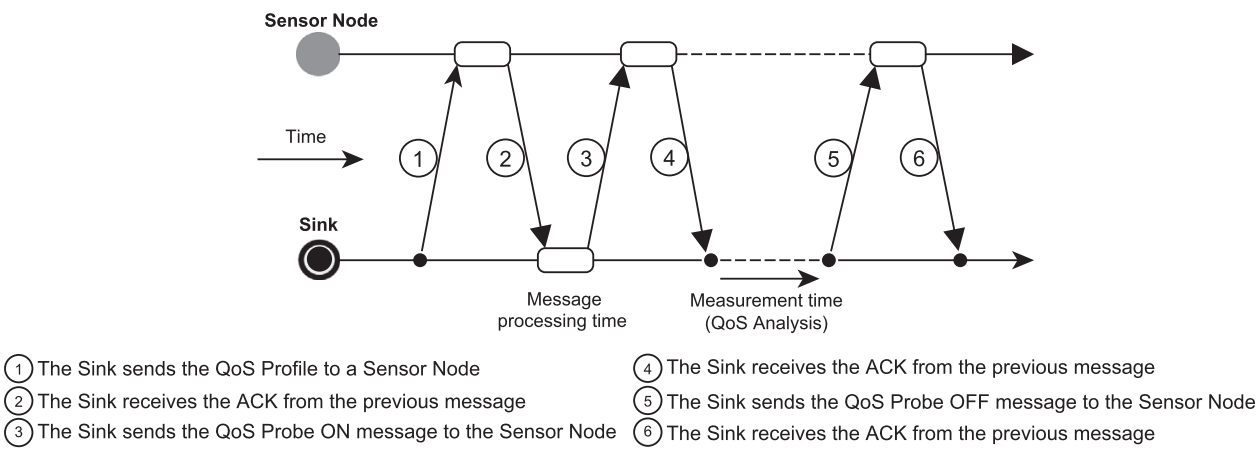

Fig. 2. Protocol implemented to detect if a new node can be admitted into the network.

\section{Proposed admission control for biomedical wireless sensor networks}

The proposed smart context-aware QoS-based admission control method uses a probe-based admission control procedure to decide where a new sensor node can join the network. The decision regarding where to admit the new sensor node on the network is based on timedomain analysis of the performance metrics used to quantify the QoS provided by the network. In this work, and to assess the performance of the proposed methodology, the Packet Reception Ratio (PRR) was the metric selected for QoS measuring purposes. Within the proposed method, the probe-based procedure uses a "virtual sensor node" called QoS Probe to mimic the presence of a new real sensor node in its neighbourhood, when necessary (e.g., on-demand or periodically triggered by software). The use of a "virtual sensor node" avoids the need of the new sensor node to be physically present within the network and enables to assess the network performance from a remote location.

\subsection{Context-awareness}

Biomedical wireless sensor networks designed to support patient monitoring applications inside hospital facilities have to face several challenges, in particular, regarding the dynamic nature of the surrounding environment. Hospital facilities can be very hostile environments for radio frequency communications. Such adversities result from structural factors such as the presence of metallic doors and furniture, as well as the existence of radiation shields (Ko et al., 2010). In fact, Ko et al. (2009) confirmed that the packet losses for IEEE 802.15.4-based radios are higher in hospitals than in other indoor environments. Moreover, IEEE 802.15.4-based networks are prone to interferences from other communication technologies such as IEEE 802.11 (i.e., Wi-Fi networks), Bluetooth devices, and cordless phones, all of which are habitually used in hospitals (Ko et al., 2010). To further worsen this scenario, low-power networks are highly vulnerable to obstacles, interferences and human bodies (Ko et al., 2010; Chen et al., 2011). Furthermore, from the previous discussion, it becomes clear that hospital environments could have a significant impact on the QoS provided by BWSNs. Moreover, the dynamic of the deployment environment affects the network performance in an unpredictable way.

The existence of such adverse effects makes clear the necessity for QoS-based admission control systems able to make decisions considering the effects of the surrounding environment (i.e., the context) on the network performance. Moreover, the context awareness is not only due to the fact that the network collects information about its operation, which is influenced by the network deployment context, but also because such information will be obtained taking into account the type of signals the new patient requires. Since the network will be probed by a node that emulates the new patient, this solution is also aware of the level of monitoring that is required (n.b., network requirements are different when different physiological signals needs to be monitored).

\subsection{Working principle of the proposed admission control method}

A common approach to control the admission of new data flows is to reserve the required bandwidth in each router that will support such data. Such solution requires a signalling protocol to preserve per-flow state, and to process per-flow reservation messages at all routers. The main drawback of that solution will be the lack of scalability, a requirement of major relevance when sensor nodes are in place. A way to avoid the above mentioned problems is to use an admission control methodology that controls the network QoS at the endpoints. Such approach uses the edge nodes to test each new admission, assessing the entire path from sink to source (Ignacio Más and Karlsson, 2007, 2008). The new proposed methodology was designed to avoid of per-hop schemes without adding any complexity to the network nodes (Abreu et al., 2014b). The proposed admission control is based on a "virtual sensor node", which is added to the network to mimic and predict the effect on the QoS when adding a new real node. Fig. 2 shows the developed methodology used to probe the network, as well all the messages exchanged to assess if the new sensor node can be admitted to the network.

The admission procedure, which is controlled by the network administrator (or who is in charge, hereinafter referred as operator), has the following phases: first the operator selects the type of sensor node to be added to the network and its preferential location in the network; then the operator requests the system to evaluate the network in order to assess its QoS; finally, based on the information retrieved by the system, the operator decides where the node can be admitted to the network. During the network evaluation phase, the system configures the QoS Probe, running on one of the sensor nodes placed in the location chosen to add the new sensor node, to mimic the presence of the new sensor node. Then, the QoS Probe starts sending a data flow, identical to that which will be generated by the real node, to the sink. Finally, the system analyses the changes in the network's QoS introduced by the new data flow and reports it to the operator that decides where the new sensor node can be admitted to the network.

\subsection{Developed software and its architecture}

The software developed to implement the proposed smart contextaware QoS-based admission control method, and to run on each node within the BWSN, was built on top of the Contiki OS (Dunkels et al., 2004) and follows the architecture presented in Fig. 3. The software developed interacts with the Contiki OS at two levels, namely at the application layer and at the network layer. This architecture facilitates the software portability to other operating systems or to be used with other communication stacks.

The software developed to implement the proposed admission control method adds the following modules to the base operating system architecture: QoS Manager, QoS Profile, QoS Probe, QoS Analyser and QoS Daemon. The QoS Manager is the interface between the application and the QoS-based network management software. It is 


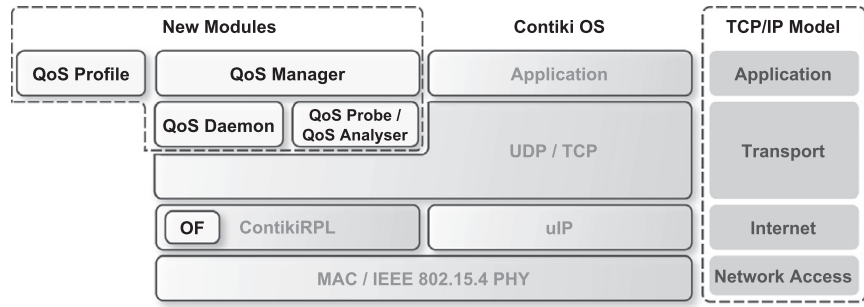

Fig. 3. Software architecture adopted to deploy the developed QoS mechanism.

responsible to manage the interactions between all the remaining components. The application uses the QoS Manager to receive information about the QoS provided by the network or to trigger actions, such as the admission control procedure. The QoS Profile contains all the relevant information about each data flow, including the required QoS. The QoS Probe (i.e., the "virtual sensor node") runs only on the sensor nodes. It can be configured, on demand, to perform remote tasks. Such tasks include generating specific data flows or collecting information about the node performance (e.g., its radio link quality). The QoS Analyser runs only in the sink node and extracts the relevant information about each data flow to assess the real-time QoS provided by the network and, if necessary, generates alerts to the healthcare information system. Finally, the QoS Daemon is in charge of all the control and signalling communications between the nodes within the BWSN.

\subsection{Foundations to use the concept of virtual sensor node}

Considering the demanding QoS requirements imposed to BWSNs, it is important to know the QoS provided by the network at any instant. This requisite is straightforward to achieve once all network nodes are within the network and running some kind of QoS monitoring software. Nevertheless, knowing the QoS being provided by the network is fundamental, it may be insufficient. In some scenarios, such as a hospital, it is necessary to anticipate the behaviour of the network before adding a new sensor node (i.e., a patient being monitored) to it, e.g., to find the most favourable location to place new patients instead of placing them in a random location potentially dangerous for the network. However, predicting the QoS that will be provided by the network after adding a new sensor node to the network is a challenging task.

Our approach to face this challenge is to postulate that we can use the deployed network to assess if a new node may be added. If we can use the network to probe itself about the consequences of adding a new node, with some specific traffic characteristics, we avoid to disrupt the network by overloading it. In this way, we propose to virtualize the sensor node that will be added to the network. Using the concept of "virtual sensor node" it is possible to assess the network without placing the new node physically in the hospital. Such node will be emulated by a real sensor node already available in the network, in the place required to be tested. However, before we proceed further with the use of a virtual node to replace the real node for test purposes, it is required to verify if both scenarios may be equivalents and what are the conditions where such approach holds.

\subsubsection{Typical scenario}

One of the key tenders of this work is that a "virtual sensor node" can simulate the presence of a real sensor node placed on its neighbourhood. In this way, the scenarios represented in Fig. 4 a) and b) may be considered equivalent. Taking into consideration the envisioned scenario (i.e., the nursing rooms inside a hospital, as pictured in Fig. 8) to use the proposed method, Fig. 4 illustrates the first-hop communications, counting from the sensor nodes to the sink. It is important to notice that, since the electromagnetic interferences existing in such scenarios are difficult to predict (e.g.,), we consider an interference-free scenario.
To mimic the behaviour of the real sensor node, the "virtual sensor node" has to generate data traffic with the same characteristics as it would be generated by the real sensor node, and both data must follow the same path to the sink. To clarify the proposed idea, let's consider Fig. 4. The left side scenario shown in Fig. 4 (i.e., Fig. 4 a)) represents a real sensor node (i.e., the $\mathrm{SN}_{\mathrm{k}}$ ) and a "virtual sensor node" (i.e., the $\mathrm{SN}_{\mathrm{i}}^{\mathrm{v}}$ ) created by the $\mathrm{SN}_{\mathrm{k}}$, the latter simulating the presence of a new sensor node on its neighbourhood. Each one of these two sensor nodes generates its own data flow, the $\mathrm{SN}_{\mathrm{k}}$ generates the data flow $\mathrm{f}_{\mathrm{k}}$ and the $\mathrm{SN}_{\mathrm{i}}^{\mathrm{v}}$ generates the data flow $\mathrm{f}_{\mathrm{i}}^{\mathrm{v}}$. In turn, the scenario represented in the right side of Fig. 4 (i.e., Fig. 4 b)) portraits the initial scenario in which the virtual sensor node was replaced by the real sensor node (i.e., the $\mathrm{SN}_{\mathrm{i}}$ ). Our proposal is that, if the data flow $\mathrm{f}_{\mathrm{i}}^{\mathrm{v}}$ as the same characteristics of the data flow $\mathrm{f}_{\mathrm{i}}$, then, those scenarios can be considered equivalent.

Looking to Fig. 4, one of the principal differences between the two scenarios presented is the different location of the sensor nodes $\mathrm{SN}_{i}^{\mathrm{v}}$ and $\mathrm{SN}_{\mathrm{i}}$. As a consequence, the data flows $\mathrm{f}_{\mathrm{i}}^{\mathrm{v}}$ and $\mathrm{f}_{\mathrm{i}}$ may reach the $\mathrm{RN}_{\mathrm{n}}$ with different delivery probabilities (n.b., the signal strength decays exponentially with respect to the distance between the nodes that are communicating. Moreover, for a given distance $d$, the signal strength is randomly distributed among the mean distance dependent value (Zuniga and Krishnamachari, 2004)). In fact, several experimental studies, considering real-world deployments, performed during the last few years have shown that wireless links can be found in one of three regions, namely connected, transitional, and disconnected (Zuniga and Krishnamachari, 2004; Nouha Baccour et al., 2012; Zamalloa and Krishnamachari, 2007). The links within the connected region are often of good quality, stable and symmetric leading to high PRR. On the other hand, the links in the disconnected region have poor quality and are inadequate to support communications (Nouha Baccour et al., 2012). Finally, within the transitional region, the links are of intermediate quality (n.b., considering a long-term evaluation), unstable, have higher coefficient of correlation in their PRRs, when compared to nearby receiver nodes located in the connected or disconnected regions, and a lot asymmetric. In view of these outcomes it is possible to make the following observations to support the proposed method:

1. Sensor nodes within the connected region have high PRRs;

2. Sensor nodes geographically close to each other, inside the connected region, have low spatial correlation in their PRRs.

Therefore, the scenarios presented in Fig. 4 can be considered equivalent if the "virtual sensor node" (i.e., the $\mathrm{SN}_{\mathrm{i}}^{\mathrm{v}}$ ) and the new real sensor node (i.e., the $\mathrm{SN}_{\mathrm{i}}$ ) are located inside of the connected region. In this way, it is of extreme importance to find the limits of the transitional region.

The authors Zuniga and Krishnamachari (2004) have identified the causes of the transitional region and have quantified their influence in the network performance. Indeed, they have derived not only expressions for the PRR as a function of the distance between the transmitter and the receiver, but also expressions to find the limits of the transitional region. Furthermore, such expressions show how the radio parameters (i.e., the modulation, the encoding, the output power at the transmitter, the noise floor, and the noise at the receiver), and the environmental parameters (i.e., the path loss exponent, the log-normal shadow variance, and the noise floor) influence the length of the transitional region. Bearing those parameters in mind, the transmitter output power is of utmost importance. It can be easily used to tune the limits of the transitional region to the needs of each specific application, taking into consideration the particular characteristics of each environment and the network deployment area. More insights about how the transmitter output power impact the beginning of the transitional region can be found in (Zuniga and Krishnamachari, 2004; Nouha Baccour et al., 2012; Zamalloa and Krishnamachari, 2007).

In view of the scenarios presented in Fig. 4 and Fig. 8, the 


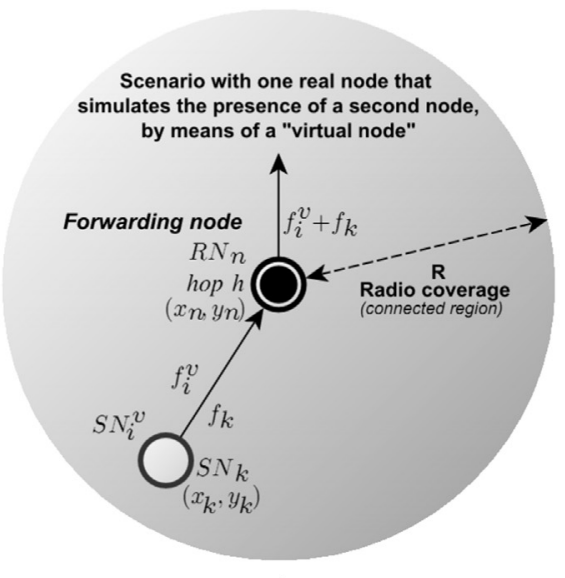

a)

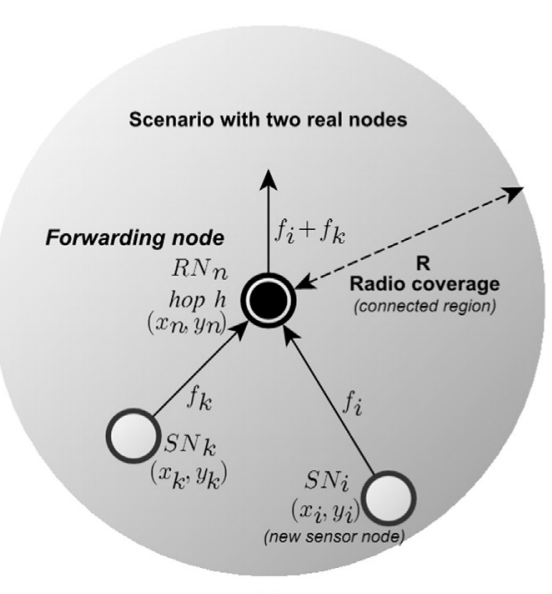

b)

Fig. 4. a) A real sensor node simulating the presence of a new sensor node, and b) the new sensor node already within the network.

connected region must have about $5 \mathrm{~m}$ in length around the receiver node (i.e., considering the dimensions of typical nursing rooms). In other words, the beginning of the transitional region must be at $5 \mathrm{~m}$ from the receiver. Moreover, considering the severe QoS requirements imposed to BWSNs a lower limit of $90 \%$ was imposed to the PRR in the connected region (n.b., this value can be adjusted according to the needs of each particular application). Thus, it is necessary to find the minimum transmitter output power necessary to fulfil those requirements.

In the following, the model presented in Zuniga and Krishnamachari (2004) will be used to find the minimum output power at the transmitter necessary to achieve the desired length for the connected region, considering the PRR limit imposed by the application. It is important to notice that this model was designed for non-coherent FSK modulation and Manchester encoding used by several radio chips (e.g., CC1000, 2005). Nevertheless, Zamalloa and Krishnamachari (2007) have shown that this model can be used as a worst case approximation for radio chips using O-QPSK modulation and DSSS encoding, e.g.: CC2420 (2011) present in the TelosB motes (Polastre et al., 2005), used in this work. Then, in order to fundament the proposed method, it will be analysed at two levels. First, at the node level, the effects of positioning the sensor nodes at different locations were investigated in view of single-hop communications. In particular, at the first hop counting from the sensor node to the sink. Then, the effects of having different delivery probabilities at single-hop communications are studied at the network level, i.e., considering multihop communications. Our analyse does not consider sensor nodes mobility nor dynamic objects inside the deployment environment, and the channel conditions for each wireless link are considered to be constant along the time, or at least during significant time intervals.

\subsubsection{Finding the output power necessary to achieve the required connected region}

The following analysis uses the log-normal shadowing path loss model (Rappapport, 2002) and the outcomes presented in Zuniga and Krishnamachari (2004) to find the minimum transmitting power, $\mathrm{P}_{\mathrm{t}}$, necessary to achieve a connected region with a radius of $5 \mathrm{~m}$. The parameters used to model the indoor environment are: path loss exponent (rate at which signal decays) $\eta=3$; standard deviation (shadowing effects) $\sigma=4$; power decay at the reference distance $d_{0}$, $\operatorname{PL}\left(\mathrm{d}_{0}\right)=55 \mathrm{~dB}$; noise floor $\mathrm{P}_{\mathrm{n}}=-105 \mathrm{dBm}$ (n.b., considering scenarios with interferences, $P_{n}$ changes over time and can be modelled as a random process (Zamalloa and Krishnamachari, 2007)); and the size of the frames used to communicate is $\mathrm{f}=70$ bytes.

Let us bound the connected region to PRRs greater than 0.9 and the transitional region to PRRs between 0.9 and 0.1 . From the theoretical model deduced in Zuniga and Krishnamachari (2004), we obtain the following SNR values for PRRs of 0.1 and $0.9, \gamma_{\mathrm{LdB}}$ and $\gamma_{\mathrm{UdB}}$, respectively,

$\gamma_{\mathrm{LdB}}=10 \log _{10}\left(-1.28 \ln \left(2\left(1-0.1 \frac{1}{8 \mathrm{f}}\right)\right)\right) \approx 7.9 \mathrm{~dB}$,

$\gamma_{\mathrm{UdB}}=10 \log _{10}\left(-1.28 \ln \left(2\left(1-0.9 \frac{1}{8 \mathrm{f}}\right)\right)\right) \approx 10.0 \quad \mathrm{~dB}$,

and we have

$\mathrm{P}_{\mathrm{t}}=\mathrm{P}_{\mathrm{n}}+\gamma_{\mathrm{U}}+\mathrm{PL}\left(\mathrm{d}_{0}\right)+2 \sigma+10 \eta \log _{10} \mathrm{~d}_{\mathrm{s}} \approx-11 \mathrm{dBm}$

for the minimum transmitting power necessary to achieve the length of $\mathrm{d}_{\mathrm{s}}=5 \mathrm{~m}$ for the connected region. In the following we analyse if a "virtual sensor node" can simulate the presence of a real sensor node placed in the connected region of $5 \mathrm{~m}$ in length.

\subsubsection{Analysing the virtual sensor node's PRR considering single-hop communications}

Consider the scenario presented in Fig. 4 without noise, i.e., $\sigma=0$. To obtain the maximum difference between the PRR achieved by the virtual data flow $\mathrm{f}_{\mathrm{i}}^{\mathrm{v}}$ and the real data flow $\mathrm{f}_{\mathrm{i}}$, considering only first-hop (i.e., the link between the $\mathrm{SN}_{\mathrm{i}}^{\mathrm{v}}$, or the $\mathrm{SN}_{\mathrm{i}}$, and the $\mathrm{RN}_{\mathrm{n}}$ ), we use the equation of PRR at a transmitter-receiver distance $d$ obtained in Zuniga and Krishnamachari (2004):

$\mathrm{p}(\mathrm{d})=\left(1-\frac{1}{2} \exp \left(-\frac{\gamma(\mathrm{d})}{1.28}\right)\right)^{8 \mathrm{f}}$,

where $\gamma(\mathrm{d})_{\mathrm{dB}}=\mathrm{P}_{\mathrm{tdB}}-\mathrm{PL}(\mathrm{d})_{\mathrm{dB}}-\mathrm{P}_{\mathrm{ndB}}, \quad \mathrm{PL}(\mathrm{d})_{\mathrm{dB}}=\mathrm{P}_{\mathrm{tdB}}-\mathrm{P}_{\mathrm{rdB}} \quad$, and $P_{r d B}$ is the received signal strength at a given distance $d$ from the transmitter. From Rappapport (2002) we have $\operatorname{PL}(\mathrm{d})=\operatorname{PL}\left(\mathrm{d}_{0}\right)+10 \eta \log _{10}\left(\mathrm{~d} / \mathrm{d}_{0}\right)+\mathrm{X}_{0, \sigma}$, where $\mathrm{X}_{0, \sigma}$ is a zero-mean Gaussian random variable (in $\mathrm{dB}$ ) with standard deviation $\sigma$ (shadowing effects). First, we obtain a relationship between $\mathrm{p}\left(\mathrm{d}_{\mathrm{i}, \mathrm{n}}\right)$ and $\mathrm{p}\left(\mathrm{d}_{\mathrm{i}, \mathrm{n}}+\Delta \mathrm{d}_{\mathrm{i}, \mathrm{n}}\right)$ for an increment $\Delta \mathrm{d}_{\mathrm{i}, \mathrm{n}}$ at the distance $\mathrm{d}_{\mathrm{i}, \mathrm{n}}$ between $\mathrm{SN}_{\mathrm{i}}$ and $\mathrm{RN}_{\mathrm{n}}$. Using the Taylor's theorem for the first-order case at the point $\mathrm{d}_{\mathrm{i}, \mathrm{n}}$, we have:

$\mathrm{p}(\mathrm{d})=\mathrm{p}\left(\mathrm{d}_{\mathrm{i}, \mathrm{n}}\right)+\dot{\mathrm{p}}\left(\mathrm{d}_{\mathrm{i}, \mathrm{n}}\right)\left(\mathrm{d}-\mathrm{d}_{\mathrm{i}, \mathrm{n}}\right)+\mathrm{R}_{\mathrm{i}, \mathrm{n}}$,

where $R_{i, n}$ is the remainder term. By properties of Eq. (1) we can state that the remainder term $R_{i, n}$ is residual in interval ]0, 5] and Eq. (2) with $R_{i, n}=0$ is a good linear approximation to Eq. (1) on ]0, 5]. Then, we have $p\left(d_{i, n}+\Delta d_{i, n}\right)-p\left(d_{i, n}\right)=\dot{p}\left(d_{i, n}\right) \Delta d_{i, n}$, for $\left.\left.d_{i, n}, d_{i, n}+\Delta d_{i, n} \in\right] 0, \quad 5\right]$, where:

$\dot{\mathrm{p}}\left(\mathrm{d}_{\mathrm{i}, \mathrm{n}}\right)=-\frac{8 \mathrm{f} \alpha \beta \eta}{2.56 \mathrm{~d}_{\mathrm{i}, \mathrm{n}}}\left(1-\frac{\alpha}{2}\right)^{8 \mathrm{f}-1}$,

$\alpha=\exp (-\beta / 1.28)$ and $\beta=10^{\left(\mathrm{P}_{\mathrm{t}}-\mathrm{PL}\left(\mathrm{d}_{0}\right)-10 \eta \log _{10}\left(\mathrm{~d}_{\mathrm{i}, \mathrm{n}}\right)-\mathrm{P}_{\mathrm{n}}\right) / 10} \approx 10^{\left(30 \log _{10}\left(\mathrm{~d}_{\mathrm{s}} / \mathrm{d}_{\mathrm{i}, \mathrm{n}}\right)+18\right) / 10}$. 
Since Eq. (3) is a decreasing non-positive function on ]0, 5], we

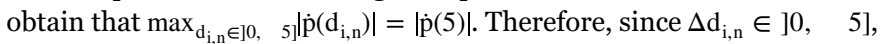
we have $\left|\mathrm{p}\left(\mathrm{d}_{\mathrm{i}, \mathrm{n}}+\Delta \mathrm{d}_{\mathrm{i}, \mathrm{n}}\right)-\mathrm{p}\left(\mathrm{d}_{\mathrm{i}, \mathrm{n}}\right)\right| \leq 1.1 \times 10^{-17} \approx 0$ in the connected region of $5 \mathrm{~m}$ in length. Thus, the difference between the PRR achieved by the virtual data flow $\mathrm{f}_{\mathrm{i}}^{\mathrm{v}}$ and the real data flow $\mathrm{f}_{\mathrm{i}}$, considering only first-hop, is approximately equal to zero. Moreover, considering shadowing effects corresponding to $\sigma=4$, by Eq. (1), we can find a sub-region with radius $d_{m} \leq d_{s}=5 \mathrm{~m}$ into the connected region of length of $5 \mathrm{~m}$ such that $\left|p\left(d_{i, n}+\Delta d_{i, n}\right)-p\left(d_{i, n}\right)\right| \approx 0$, for $\left.\left.d_{i, n}, d_{i, n}+\Delta d_{i, n} \in\right] 0, \quad d_{m}\right]$. So, we also conclude that the virtual data flow is approximately equal to real data flow inside this sub-region with radius $\mathrm{d}_{\mathrm{m}}$. If one of the two sensor nodes $\mathrm{SN}_{\mathrm{i}}^{\mathrm{v}}$ or $\mathrm{SN}_{\mathrm{i}}$ is out of this sub-region, we obtain $\left|p\left(d_{i, n}+\Delta d_{i, n}\right)-p\left(d_{i, n}\right)\right| \leq 0$. 1, i.e., the maximum difference between the PRR achieved by the virtual data flow $\mathrm{f}_{\mathrm{i}}^{\mathrm{v}}$ and the real data flow $\mathrm{f}_{\mathrm{i}}$ is 0.1 . However, both have a rate greater than or equal to $90 \%$.

\subsubsection{Analysing the virtual sensor node's PRR considering multi-hop communications}

Rather than single-hop communications, typical BWSNs have multiple hops between the source sensor nodes and the sink. In fact, they may have not only several hops between those nodes but also several paths to route the data gathered by the sensor nodes to the sink. In that way, two sensor nodes can send their data to the sink using distinct data paths, each one having a particular PRR associated. In our proposal we consider that the data flow $\mathrm{f}_{\mathrm{i}}^{\mathrm{v}}$, generated by the "virtual sensor node" $\mathrm{SN}_{\mathrm{i}}^{\mathrm{v}}$ of Fig. 4a), follows the best path to reach the sink (n.b., the rule to find the best path depend on the routing protocol in use). Moreover, the sensor node $\mathrm{SN}_{\mathrm{i}}$ will use the same path to send its data to the sink. In other words, we are assuming that the "virtual sensor node", $\mathrm{SN}_{\mathrm{i}}^{\mathrm{v}}$, and the real sensor node, $\mathrm{SN}_{\mathrm{i}}$, use the same data path to send their data to the sink. In this way, the PRR associated with the path between the $\mathrm{RN}_{\mathrm{n}}$ and the sink is the same for both data flows. Additionally, as it was shown in the last subsection, the PRRs achieved by the virtual data flow $f_{i}^{v}$ and the real data flow $f_{i}$ at the first hop are very similar (with a maximum error of $10 \%$ ). Consequently, it is possible to argue that the PRRs of both data flows, for the entire path (i.e., from the sensor node until the sink) are almost equivalent.

In summary, our proposal is that a "virtual sensor node" can produce an equivalent effect in the performance of a WSN, when compared with a real sensor node, if both sensor nodes produce data with the same characteristics, both data flows follow the same path to the sink, and they are within the connected region experiencing the same radio conditions.

\subsubsection{Experimental validation}

Bearing in mind the previous requirements, the following experiment was performed in a real world scenario recreating the conditions existing in a nursing room (e.g., with fixed obstacles and metal furniture), as follows: a sink node and a sensor node were used to measure the PRR at different distances between the two nodes and for several angles all around the receiver as pictured in Fig. 5, in order to find the minimum transmitter power necessary to achieve a PRR of, at least, $90 \%$ inside the connected region and set the beginning of the transitional region to $5 \mathrm{~m}$ from the transmitter. Assessing the PRR in different directions is crucial since the radiation pattern of the antenna is anisotropic due to the fact that the antennas have different gains at different propagation directions, resulting in different PRRs (Nouha Baccour et al., 2012). Therefore, it is necessary to tune the output power of the network nodes in order to ensure the desired connected region in all directions. The PRR for each pair (distance, angle) was calculated after the sensor node had sent five hundred messages to the receiver.

The results obtained from such experiment are pictured in Fig. 5. Considering such results it is possible to argue that the minimum output power at the transmitter necessary to achieve a connected region of $5 \mathrm{~m}$ in length, with a PRR of, at least, $90 \%$, is $-10 \mathrm{dBm}$, as predict earlier by the analytic model. It is important to notice that these results depend on each specific environment and network deployment area. Consequently, these results must be tuned for each network deployment.

\section{Methodology assessment in a real hospital environment}

Real-world experiments were performed to evaluate the smart context-aware QoS-based admission control method. The experiments were made in a small-sized hospital situated in Esposende, a small city near Braga, Portugal.

\subsection{Preliminary findings and initial deployment}

The deployment of BWSNs in real-world environments is a challenging task even to engineers with high levels of expertise in BWSNs systems. Moreover, when harsh environments are at stake, such as hospital facilities or nursing homes, the difficulties to get BWSNs working properly worsens. Hence, each real-world BWSN deployment must be carefully designed not merely according to the demands of each target application, but also considering the particular characteristics of each deployment area.

\subsubsection{Preliminary findings}

Based on the knowledge obtained from this particular deployment, it is possible to argue that each real-world deployment is unique. More, it is strictly necessary to study and understand the intrinsic characteristics of each deployment site, namely those that can compromise the quality of the wireless channel, such as: attenuation of the radio signal caused by metallic furniture, interferences caused by other medical equipment's, and other negative effects caused by peoples' routines (e.g., staff shifts or visiting times). Such study is vital to design a suitable network deployment strategy in order to achieve the required performance.

Regarding the deployment scenario used to evaluate the proposed admission control method, the following procedure was made in order to understand the intrinsic characteristics of the network deployment site. First, information was collected about the use made of the network deployment area. Second, an inspection was made to identify other radio communication infrastructures (e.g., an IEEE802.11 network) existing on the network deployment area. Third, an examination was made to find hospital furniture and/or medical equipment able to cause attenuation/interferences in the radio signals (e.g., fading due to multipath or due to shadowing from obstacles). Finally, information was requested about the hospital staff shifts and about the visiting schedule.

It is important to emphasise that the research team had no control on the environment conditions, or on the use of any hospital/medical equipment, during the field tests. All the tests were made under the supervision of the hospital staff.

The area used to perform the field tests (i.e., the shaded area presented in the Fig. 6) has a nursing desk and three nursing rooms in which low-acuity patients stay for a period that, typically, do not exceed eight days. Those nursing rooms are not private, if necessary two or three patients can share the same room.

From our survey, it was found that the deployment area does not have any other wireless communication technology. Instead, the information system uses a wired communication infrastructure. Therefore, it was not necessary to perform any tests to study the radio activity in the deployment area. When other wireless communication technologies are detected, it is necessary to study the coexistence of both technologies, as explained by in Ko (2012).

Concerning the signal propagation and electromagnetic interferences (or any other effect able to deteriorate the radio signal), neither the hospital furniture nor the medical equipment found in the deployment area is susceptible of causing significant damages to the 


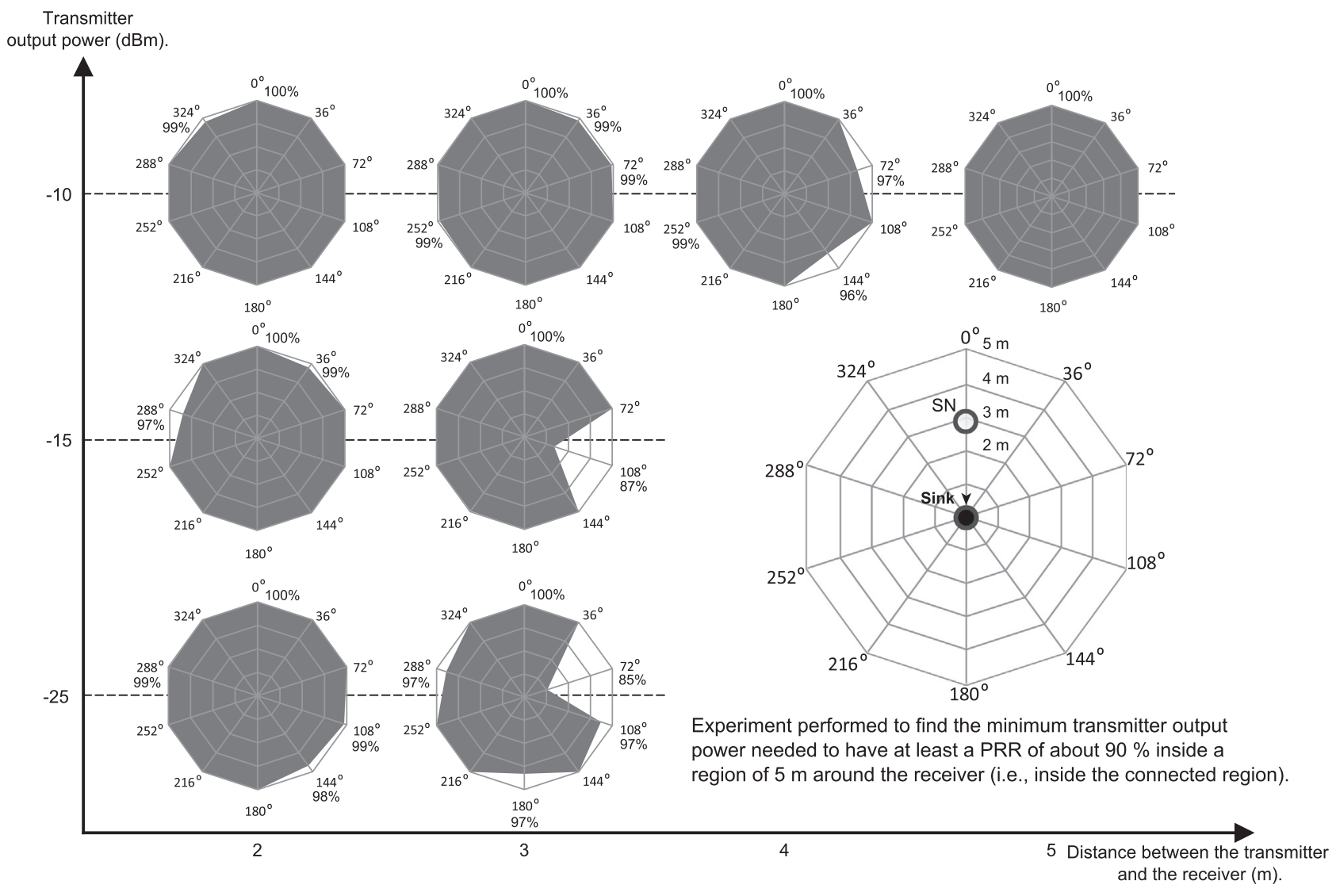

Fig. 5. The PRR as a function of the transmitter output power, the distance and angle between the sender and the receiver.

radio channel. On its turn, the staff daily routines can be classified as regular concerning the normal activities of a non-acute hospital area. So, on this particular deployment area, the radio signal propagation is affected only by the building structure (e.g., walls, corridors or steel doors) and by the human bodies present on the site. During the field tests the average amount of people inside of the network deployment area, simultaneously, did not exceed a dozen. So, the network deployment area can be considered as having low density of people.

\subsubsection{Initial deployment}

After the preliminary study just described, it was necessary to design the network deployment strategy. First, the network physical topology was defined. Following the strategy proposed by the MEDiSN project (Ko et al., 2010a, 2010b), the solution adopted makes use of a backbone of Relay Nodes (RNs) and several Sensor Nodes (SNs), defining a tree topology as represented in the in Fig. 7. As explained by the authors of the MEDiSN project, the backbone is used both to form a

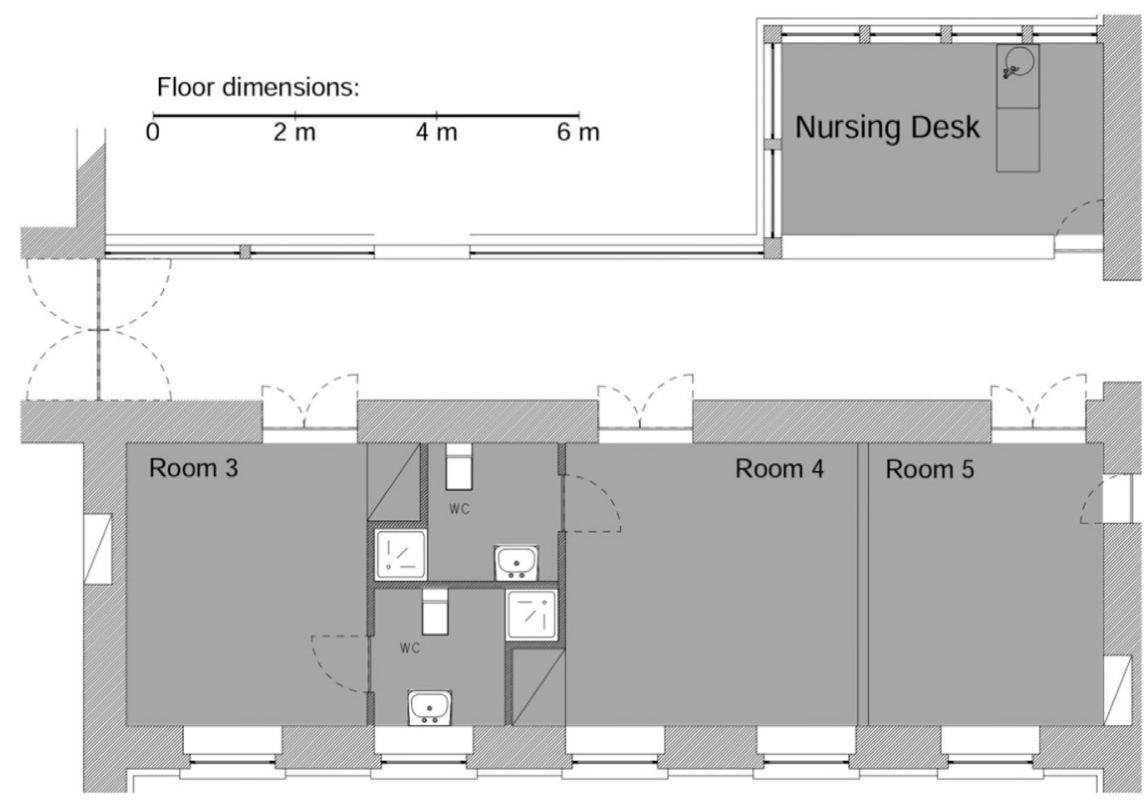

Fig. 6. Floor plan of the Hospital showing the nursing rooms and the nursing desk used to deploy the BWSN. 


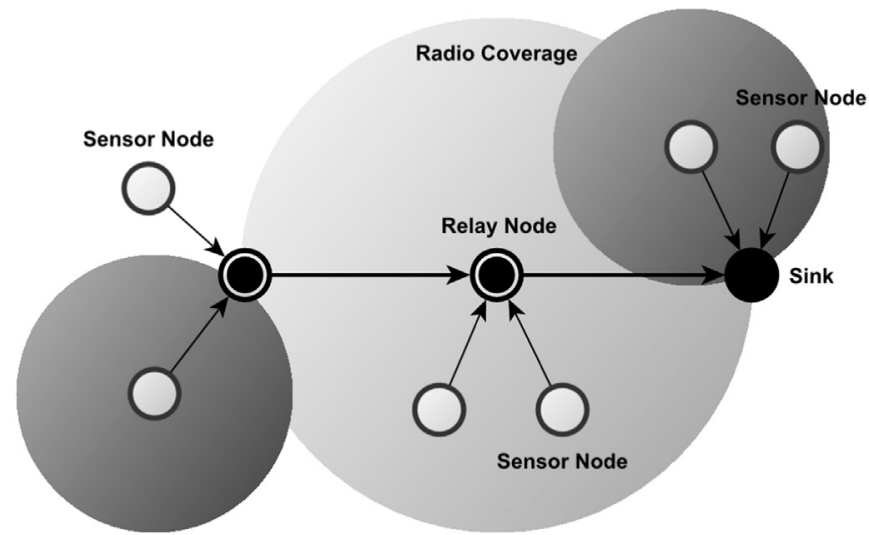

Fig. 7. The network topology makes use of a backbone of relay nodes to ensure the necessary coverage and to route the data packets to the sink.

bidirectional link to the Sink and to ensure the required network coverage. Moreover, the use of a backbone has several benefits. It allows expanding the network, both to improve its performance (e.g., by carefully placing more RNs in the same area) and to increase the network coverage (i.e., improving the network scalability).

Since the nursing staff will use information retrieved by the proposed smart context-aware QoS-based admission control method to make a decision about the best location to place the patients, the natural location to place the sink is the nursing desk, so it can be connected to the computer running the frontend interface of the network management system. The backbone contains two RNs strategically placed to ensure good connectivity inside the nursing rooms three and four.

Fig. 8 shows the deployment of both the sink and the backbone (n.b., the links between the RNs within the backbone and between the RNs and the sink are merely for example purposes. In reality, the backbone is self-organized).

Several tests were made in order to determine the best spots to set up the RNs, while ensuring both the necessary network coverage and the required network performance. To assess the network's performance the end-to-end PRR was used as a figure-of-merit. Based on the tests performed the RNs were deployed as shown in the Fig. 8. After deploying the RNs backbone, the BWSN became fully functional and ready to admit the patients, i.e. the sensor nodes.

\subsection{Experiments}

In the following all the experiments performed to evaluate the smart context-aware QoS-based admission control method are explained in detail, in particular the use of a "virtual sensor node" to mimic the presence of a new real sensor node within the network.

The evaluation procedure was made in two distinct scenarios, according to the number of nursing rooms used and patients being monitored. By using different nursing rooms, it was possible to assess the proposed method taking into account the impact of the hospital structure (e.g., granite walls, corridors and doors) on the propagation of the radio signals and, consequently, on the network performance. On its turn, the use of several patients makes the experiments closer to reality, assessing the suggested method under realistic conditions.

In the first scenario, no more than three patients were monitored while sharing the same nursing room (i.e., the room 4 from Fig. 8). This scenario was designed to assess the proposed method without interferences from patients being monitored in the neighbourhood and, at the same time, experiencing the minimum impact from the deployment environment. By using this scenario, the proposed method will be used to assess the impact of adding a new patient to the network.

Then, in the second scenario, a maximum of five patients were monitored while distributed by two non-adjacent nursing rooms (i.e., the rooms 3 and 4, separated by two bathrooms, as pictured in Fig. 8). This scenario was designed to assess the suggested QoS-based admission control method under interferences from patients being monitored in the neighbourhood of the "virtual sensor node". Moreover, this scenario is also useful to study the impact of using the proposed method, on the patients being monitored in the neighbourhood of the "virtual sensor node".

These scenarios were designed with the purpose to verify the following hypothesis:

Hypothesis. Adding a "virtual sensor node" to a BWSN produces an equivalent impact in the network's performance, when comparing with the addition of a new real sensor node. H. 1.

If this hypothesis was confirmed, the proposed smart context-aware QoS-based admission control method can be used to answer the following questions:

- Which is the impact of adding a new patient to a BWSN, in the QoS being provided to the patients already within the network?

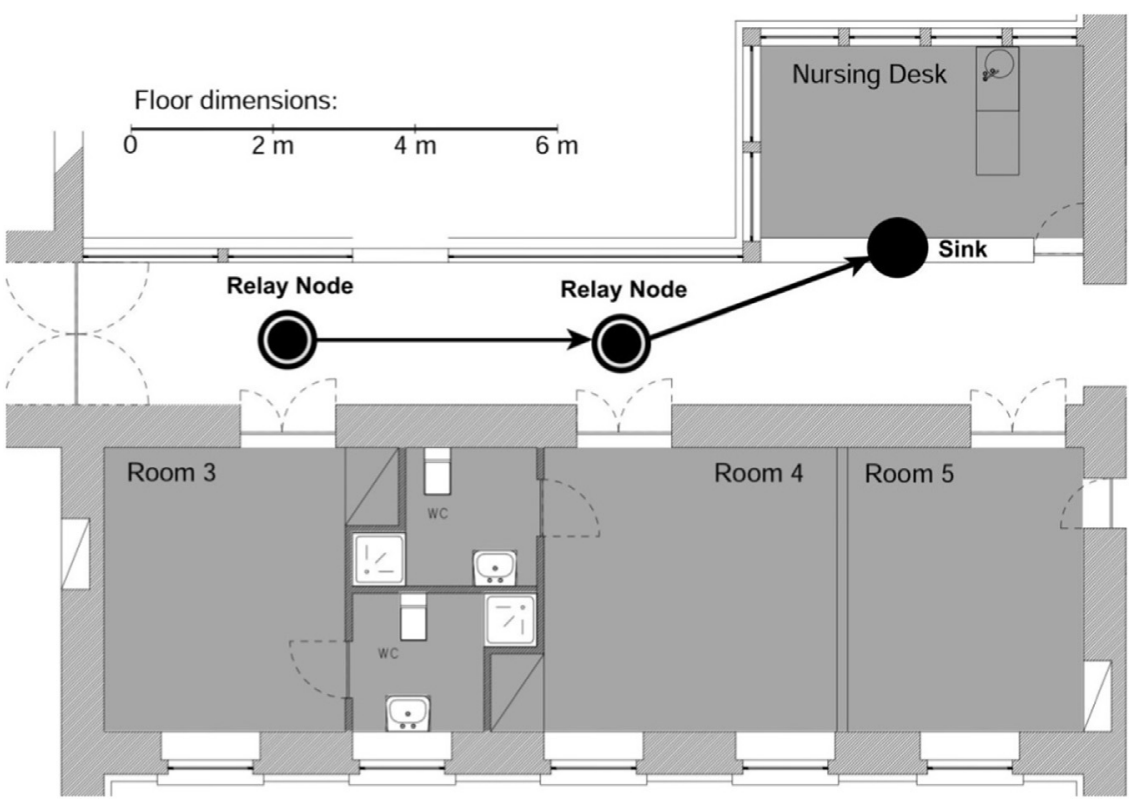

Fig. 8. Initial deployment, showing the relay nodes forming a backbone to the sink. 
- Which is the best location to add a new patient to a BWSN, while minimising its impact in the QoS being provided by the network?

In such context, all the experiments were performed under the following assumptions and constraints:

- The term patient is only a nomenclature used to improve the understanding of the experiments. In reality, they are represented by the sensor nodes;

- The physiological data are generated by the sensor nodes;

- The data generated by the "virtual sensor node" and by the new patient follow the same path to the sink.

- The mobility of the sensor nodes is very limited and unable to cause changes in the network topology (i.e., in both the physical and logical topologies).

Regarding the patient monitoring application used to perform the experiments, its purpose is to monitor three vital signs (i.e., temperature, heart rate, and respiratory rate) and the oxygen saturation (SpO2). To do so, each sensor node generates data traffic according to the characteristics of each one of these signals, resulting in an aggregated data rate of about $1 \mathrm{kbps}$. For a detailed description of the application and network configurations, see Table 1 .

Regarding the results obtained from the experiments, they were analysed considering not only all the stages of the admission control procedure, but also the results obtained when adding the new real sensor node to the BWSN. Regarding the admission control procedure, two different time intervals were considered: the time interval from the beginning of the experiment until the instant when the QoS Probe is turned ON, denoted as: $\mathrm{t} \in] 0, \mathrm{t}_{\text {QoS Probe ON }}$ ]; and the time interval in which the QoS Probe is sending data packets to the sink, denoted as: $t \in] t_{\text {QoS Probe ON }}, \quad t_{\text {QoS Probe OFF }}$. After the admission control procedure, two new intervals were investigated, namely: the time interval between the end of the admission procedure and the instant when the new real sensor node is admitted to the BWSN, denoted as: $\left.\mathrm{t} \in] \mathrm{t}_{\mathrm{QoS} \text { Probe OFF }}, \mathrm{t}_{\text {New SN ON }}\right]$; and the time interval in which the new real sensor node is sending data packets to the sink, denoted as: $\left.\mathrm{t} \in \mathrm{t}_{\text {New SN ON }}, \quad \mathrm{t}_{\text {New SN OFF }}\right]$.

During the following experiments the PRR is taken as figure-ofmerit to evaluate the QoS provided by the network. The PRR of each sensor node was defined as:

$\operatorname{PRR}_{\mathrm{i}}=\frac{\mathrm{R}_{\mathrm{i}}}{\mathrm{S}_{\mathrm{i}}}$

Table 1

Application and network level configurations common to all the experiments.

\begin{tabular}{|c|c|}
\hline \multicolumn{2}{|l|}{ Application: } \\
\hline Operating System & Contiki OS \\
\hline Task type & Time driven \\
\hline Data length & 70 bytes (per packet) \\
\hline Data reporting interval & $500 \mathrm{~ms}$ \\
\hline QoS-probe reporting interval & $500 \mathrm{~ms}$ \\
\hline \multicolumn{2}{|l|}{ Network: } \\
\hline Number of sinks & 1 \\
\hline Number of relay nodes & 2 \\
\hline Number of sensor nodes & $\begin{array}{l}3,4,5 \text { (depending on the scenario being } \\
\text { assessed) }\end{array}$ \\
\hline Transmission power & $\begin{array}{l}0 \mathrm{dBm} \text { (in both, the sink and the RNs), }-7 \mathrm{dBm} \\
\text { or }-10 \mathrm{dBm} \text { (in the SNs) }\end{array}$ \\
\hline Network Layer & IPv6 with 6LowPAN \\
\hline Transport Layer & UDP \\
\hline Routing protocol & $\begin{array}{l}\text { RPL - DIO minimum interval } 65 \mathrm{~s} \text { and DIO } \\
\text { maximum interval } 70 \mathrm{~min}\end{array}$ \\
\hline \multicolumn{2}{|l|}{ Experiments: } \\
\hline Time & Between 90 and 120 min \\
\hline
\end{tabular}

where the $P R R_{i}$ is the PRR of the sensor node $S_{i}, R_{i}$ is the number of data packets received by the sink from the $\mathrm{SN}_{\mathrm{i}}$, and $\mathrm{S}_{\mathrm{i}}$ is the number of data packets sent by the $\mathrm{SN}_{\mathrm{i}}$.

\subsubsection{Network deployment area covering one nursing room}

This scenario, i.e., monitoring a maximum of three patients sharing the same nursing room, was designed to verify if the proposed admission control method could be used to answer the following question:

Question. Is it possible to use the concept of "virtual sensor node" to estimate the QoS impact of adding a new patient to a BWSN, considering that all the sensor nodes share the same physical space? Q. 1.

Seeking the answer for this question, the following scenario, comprising two patients being monitored while sharing the same physical space (i.e., the same nursing room), was designed.

In such case, the proposed smart context-aware QoS-based admission control method can be used to investigate the QoS impact when adding a third patient to the BWSN, while sharing the same physical space with the other two patients. Finally, considering the results retrieved by the admission control method, it is possible to decide about where to locate a new patient.

Considering this particular scenario, and the network logical topology represented in Fig. 9, the results obtained by using the proposed admission control method to assess the BWSN and decide about the admission of a new patient are summarised in Table 2.

Initially the network was being used to carry the data generated by two patients (i.e., the $\mathrm{SN}_{1}$ and the $\mathrm{SN}_{2}$ ). In such case, the network can be considered highly efficient and providing high standards of QoS. Such fact can be confirmed by analysing the PRR of the data flows generated by both sensor nodes, during the time periods: $\left.t \in] 0, t_{\text {QoS Probe ON }}\right]$ and $\left.t \in] t_{\text {QoS Probe OFF }}, t_{\text {New SN ON }}\right]$. To be precise, during these two time intervals, the $\overline{\mathrm{PRR}}$ of the data flow generated by the $\mathrm{SN}_{1}$ is of about 99. $1 \%$ and $98.9 \%$, respectively. On its turn, the $\overline{\mathrm{PRR}}$ related to the data flow generated by the $\mathrm{SN}_{2}$, during the same time intervals, is of about $98.9 \%$ and 98. 2\%, respectively. Then, when using the QoS Probe to assess the possibility of adding a new patient to the network, sharing the same nursing room, which comprises the interval $\left.t \in] t_{\text {QoS Probe ON }}, t_{\text {QoS Probe OFF }}\right]$, the PRR of the data flows generated by both the $\mathrm{SN}_{1}$ and the $\mathrm{SN}_{2}$ drops considerably. Indeed, the $\overline{\mathrm{PRR}}$ of the data flow generated by the $\mathrm{SN}_{1}$ drops about $9.7 \mathrm{pp}$ from $99.1 \%$ to $89.4 \%$ and the $\overline{\mathrm{PRR}}$ of the data flow generated by the $\mathrm{SN}_{2}$ drops from about $98.9 \%$ to $90.6 \%$, corresponding to a degradation of about $8.3 \mathrm{pp}$. On its turn, the $\overline{\text { PRR }}$ achieved by the QoS Probe is around 92\%, which is comparable with the values achieved by the other sensor nodes. Such degradation in the PRR of both data flows can be justified by the additional traffic generated by the QoS Probe. In fact, such additional traffic makes the competition for the transmission medium more difficult, increasing the probability of collisions as well as the interferences experienced by each sensor node, not only due to the presence of the QoS Probe within the network but also due to the additional retransmissions performed by the $\mathrm{RN}_{1}$.

Finally, after the admission of the $\mathrm{SN}_{3}$ (i.e., the new patient), that corresponds to the time interval $\left.t \in] \mathrm{t}_{\mathrm{New} \mathrm{SN} \mathrm{ON}}, \mathrm{t}_{\mathrm{New} \mathrm{SN} \mathrm{OFF}}\right]$, the $\overline{\text { PRR }}$ of the data flows generated both by the $\mathrm{SN}_{1}$ and by the $\mathrm{SN}_{2}$ falls about 3. $6 \mathrm{pp}$, from $98.9 \%$ to $95.3 \%$, and $4.6 \mathrm{pp}$, from $98.2 \%$ to $93.6 \%$, respectively. On its turn, the $\overline{\mathrm{PRR}}$ of the data flow generated by the $\mathrm{SN}_{3}$ is of about $94.1 \%$, which is comparable with the PRR achieved by the other sensor nodes. In that case, the degradation on the PRR can also be explained using the arguments presented earlier. However, since the data generation processes are not time synchronised, the impact of adding the sensor nodes (i.e., the QoS Probe and the $\mathrm{SN}_{3}$ ) in different moments affects the performance of the network in diverse ways.

To that end, it is necessary to compare the PRR achieved during 


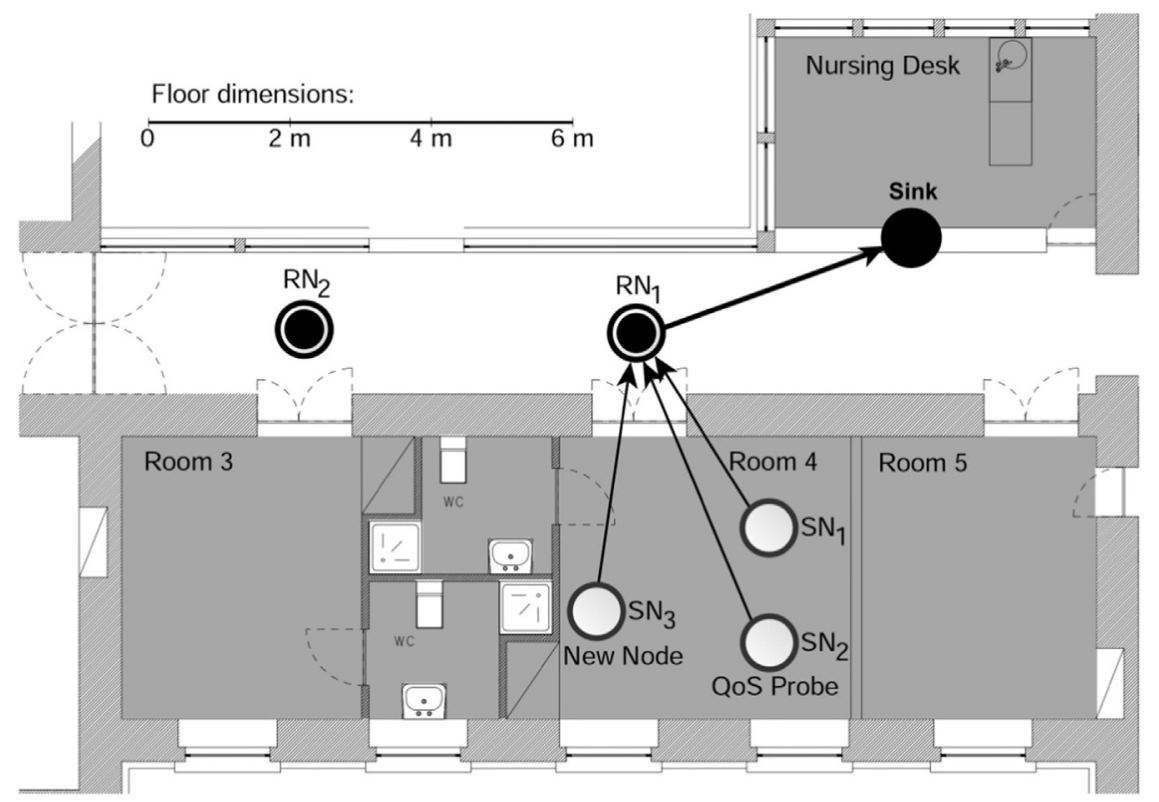

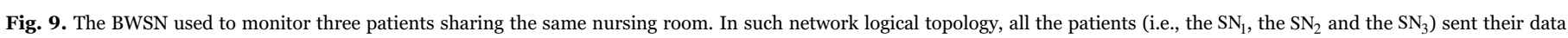
packets to the sink through the backbone. The network topology is pictured according the sink's routing table.

those time intervals. So, to study the similarity between the PRRs of each data flow during the assessment period and after adding the new sensor node to the network, the percentage difference was used:

$\mathrm{PD}=200\left|\frac{\mathrm{PRR}_{\mathrm{x}}-\mathrm{PRR}_{\mathrm{y}}}{\mathrm{PRR}_{\mathrm{x}}+\mathrm{PRR}_{\mathrm{y}}}\right|$,

where the $\mathrm{PRR}_{\mathrm{x}}$ and $\mathrm{PRR}_{\mathrm{y}}$ represent the PRRs being studied.

Considering the percentage difference between the $\overline{P R R}$ of each sensor node regarding the time periods, $\left.t \in] t_{\text {QoS Probe ON }}, t_{\text {QoS Probe OFF }}\right]$ and $\left.t \in] t_{\text {New SN ON }}, \quad t_{\text {New SN OFF }}\right]$, as shown in Table 2, it is possible to verify that, considering this experiment, the impact of adding a "virtual sensor node" to the network is comparable (i.e., with a maximum average difference of about 6.4\%) to the impact of adding a real sensor node to the network. Moreover, considering the percentage difference between the $\overline{\text { PRR }}$ of each sensor node after the admission of the new patient and the $\overline{\mathrm{PRR}}$ achieved by the QoS Probe, it is possible to conclude that the $\overline{\text { PRR }}$ of each sensor node after adding the new sensor node to the network is equivalent (i.e., with a maximum average difference of about 3.5\%) to the one achieved by the QoS Probe. Considering these results, the proposed smart context-aware QoSbased admission control method was able to predict the impact of adding the new patient to the network.

The results obtained allow us to conclude that the response to the question formulated in the beginning of this experiment (i.e., the question Q. 1) is affirmative. Moreover, in the context of the present scenario, the hypothesis H. 1 was confirmed to be true.

\subsubsection{Network deployment area covering two nursing rooms}

Regarding the second scenario, i.e., monitoring a maximum of five patients spatially distributed into two non-adjacent nursing rooms (i.e., the rooms 3 and 4 from Fig. 10), it was designed to verify if the proposed admission control method can be used to answer the following question:

Question. Is it possible to use the concept of "virtual sensor node" to estimate the QoS impact when adding a new patient to a BWSN, if the new patient does not share the same physical space with all the others patients within the network? Q. 2.

Among all the experiments performed using this scenario, the following was chosen to be discussed in order to find the response to the question Q. 2.

Regarding this experiment, the $\mathrm{SN}_{1}$ sends its data to the sink through the $\mathrm{RN}_{2}$ while the remaining sensor nodes, including the QoS Probe and the new sensor node (i.e., the $\mathrm{SN}_{4}$ ), use the $\mathrm{RN}_{1}$ to forward their data packets to the sink (see Fig. 10 for more details). In this case, the data generated by the QoS Probe and by the new sensor node follow the same path to reach the sink. Unlike the former experiment, in this case it is likely that the QoS Probe and the new sensor node achieve comparable performance in terms of PRR. The results obtained when assessing the current scenario, using the network topology pictured in Fig. 10 and explained in detail in Table 3.

Considering the results presented in Table 3 , it is possible to verify that during both the assessment period using the QoS Probe and the period next to the introduction of the new sensor node, the network's performance suffers a significant degradation. Moreover, it is important to notice that the performance degradation experienced by all the sensor nodes inside the room 4 is comparable, including the QoS Probe and the new sensor node (i.e., the $\mathrm{SN}_{5}$ ). This outcome was expected since the data generated both by the QoS Probe and by the $\mathrm{SN}_{5}$ use the

Table 2

Results obtained when assessing the BWSN considering the network topology presented in Fig. 9.

\begin{tabular}{|c|c|c|c|c|c|c|}
\hline \multirow[t]{2}{*}{ Network Running Time (t) } & \multicolumn{6}{|c|}{ Packet Reception Ratio $\left(\overline{\mathbf{P R R}}_{ \pm \sigma}\right) \quad \%$} \\
\hline & $\mathrm{SN}_{1}$ & & $\mathrm{SN}_{2}$ & & QoS Probe & New $\mathrm{SN}\left(\mathrm{SN}_{3}\right)$ \\
\hline $\left.\mathrm{t} \in] 0, \quad \mathrm{t}_{\mathrm{QoS} \text { Probe ON }}\right]$ & 99.1 & \pm 0.9 & 98.9 & \pm 1.1 & n.a. & n.a. \\
\hline $\mathrm{t} \in] \mathrm{t}_{\mathrm{QoS}}$ Probe ON,$\left.\quad \mathrm{t}_{\mathrm{QoS} \text { Probe OFF }}\right]$ & 89. 4 & \pm 4.4 & 90. 6 & \pm 5.0 & 92. $0 \pm 2.7$ & n.a. \\
\hline $\mathrm{t} \in] \mathrm{t}_{\mathrm{QoS} \text { Probe OFF }}, \quad \mathrm{t}_{\mathrm{New}} \mathrm{SN}$ ON $]$ & 98.9 & \pm 1.1 & 98.2 & \pm 1.8 & n.a. & n.a. \\
\hline $\left.\mathrm{t} \in] \mathrm{t}_{\text {New SN ON }}, \quad \mathrm{t}_{\text {New SN OFF }}\right]$ & 95.3 & \pm 2.0 & 93. 6 & \pm 4.3 & n.a. & $94.1 \pm 4.2$ \\
\hline
\end{tabular}




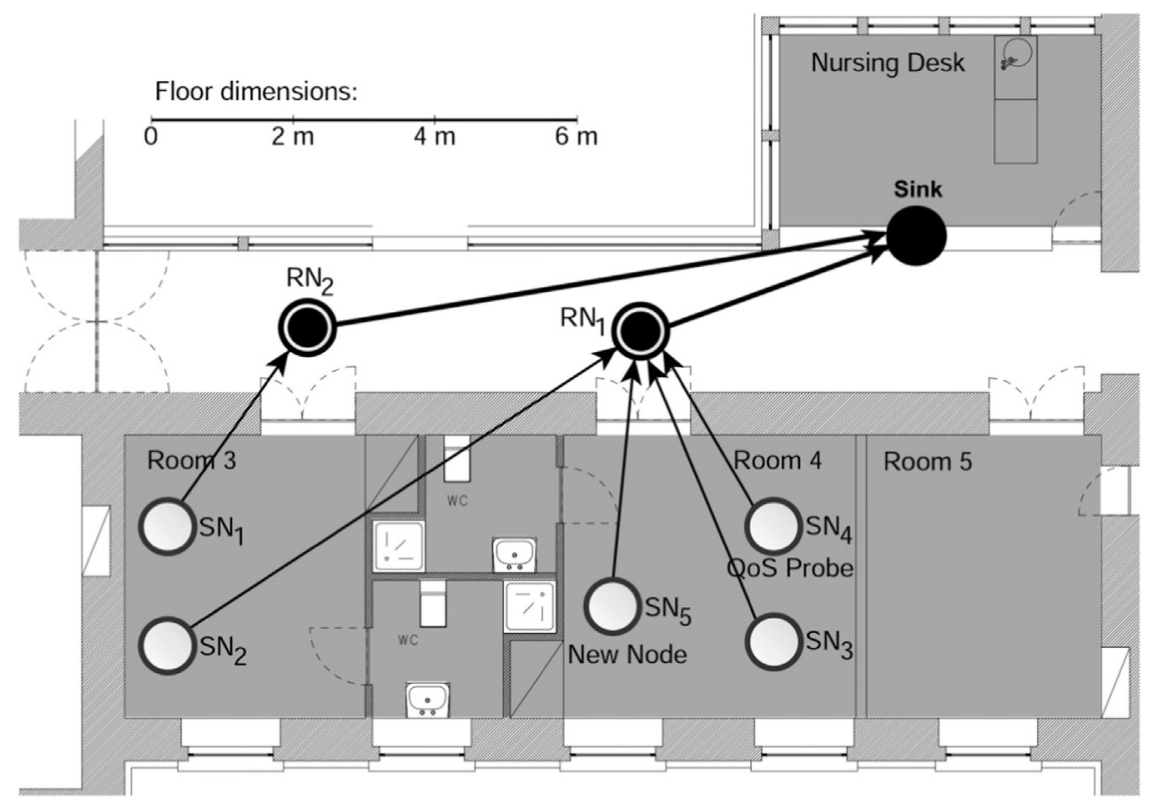

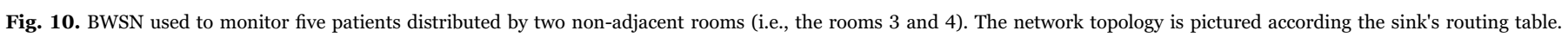

Table 3

Results obtained when assessing the BWSN, considering the network topology presented in Fig. 10.

\begin{tabular}{lcccccc}
\multirow{2}{*}{ Network Running Time $(\mathrm{t})$} & \multicolumn{5}{c}{ Packet Reception Ratio $(\overline{\text { PRR }} \pm \mathbf{\sigma}) \%$} \\
\cline { 2 - 6 } & $\mathrm{SN}_{1}$ & $\mathrm{SN}_{2}$ & $\mathrm{SN}_{3}$ & $\mathrm{SN}_{4}$ & QoS Probe & New SN $\left(\mathrm{SN}_{5}\right)$ \\
\hline $\mathrm{t} \in\left[0, \mathrm{t}_{\text {QoS Probe ON }}\right]$ & $83.9 \pm 4.4$ & $83.5 \pm 4.1$ & $86.2 \pm 3.1$ & $86.3 \pm 3.3$ & n.a & n.a \\
$\mathrm{t} \in\left[\mathrm{t}_{\text {QoS Probe ON }}, \mathrm{t}_{\text {QoS Probe OFF }}\right]$ & $\mathbf{5 7 . 1} \pm \mathbf{7 . 3}$ & $\mathbf{5 7 . 1} \pm \mathbf{7 . 3}$ & $\mathbf{6 3 . 5} \pm \mathbf{9 . 5}$ & $\mathbf{6 5 . 7} \pm \mathbf{7 . 7}$ & $\mathbf{6 3 . 7} \pm \mathbf{6 . 7}$ & n.a \\
$\mathrm{t} \in\left[\mathrm{t}_{\text {QoS Probe OFF }}, \mathrm{t}_{\text {New SN ON }}\right]$ & $86.8 \pm 2.5$ & $86.3 \pm 3.2$ & $82.5 \pm 8.4$ & $80.6 \pm 8.8$ & n.a & n.a \\
$\left.\mathrm{t} \in] \mathrm{t}_{\text {New SN ON }}, \mathrm{t}_{\text {New SN OFF }}\right]$ & $\mathbf{6 4 . 5} \pm \mathbf{7 . 0}$ & $\mathbf{6 5 . 1} \pm \mathbf{8 . 5}$ & $\mathbf{6 6 . 9} \pm \mathbf{9 . 6}$ & $\mathbf{6 7 . 1} \pm \mathbf{8 . 3}$ & n.a & $\mathbf{6 5 . 9} \pm \mathbf{6 . 8}$ \\
\hline
\end{tabular}

same path to reach the sink. By using the same data path, it is expected that both have the comparable impact on the performance of the sensor nodes on their neighbouring (i.e., inside the room 4).

Comparing the $\overline{\mathrm{PRR}}$ of all the sensor nodes within the network during the network assessment period (i.e., $\left.t \in] \mathrm{t}_{\mathrm{QoS} \text { Probe ON }}, \mathrm{t}_{\mathrm{QoS} \text { Probe OFF }}\right]$ ) and the period after the admission of the new sensor node to the network (i.e., $\left.t \in] t_{\text {New SN ON }}, \quad t_{\text {New SN OFF }}\right]$ ) using the percentage difference as defined in Eq. (4), it is possible to conclude that both the QoS Probe and the new sensor node affect the sensor nodes within the network distinctly. In fact, looking to the results of Table 3 it is clear that the performance of the sensor nodes inside the room 3 (i.e., the $\mathrm{SN}_{1}$ and the $\mathrm{SN}_{2}$ ) are distinctly affected by the QoS Probe and by the new sensor node. Nevertheless, the sensor nodes inside the room 4 (i.e., the $\mathrm{SN}_{3}$ and the $\mathrm{SN}_{4}$ ) have seen their performance affected in a comparable way by the QoS Probe and by the new sensor node. Moreover, when comparing the PRR achieved by each sensor node within the network, including the new one, with the $\overline{\text { PRR }}$ achieved by the QoS Probe during its operation, it is possible to verify that the $\overline{\text { PRR }}$ of all the sensor nodes after the admission of the new sensor node is comparable with the one achieved by the QoS Probe. In view of these results it is possible to argue that the proposed method was able to predict the impact of adding the new node to the network.

Regarding the results obtained during the aforementioned experiment, it is possible to claim that the proposed QoS-based admission control method was able to predict the impact of adding the new patient to the network. So, the response to the question Q. 2 is affirmative and the hypothesis H. 1 is considered to be true.

Considering the results found from the previous experiments, under the previously stated assumptions and constraints, it is possible to argue that, the proposed QoS-based admission control method can be used to predict the impact of adding a new sensor node to a BWSN. Moreover, applying this method to different locations, manually or using an autonomous process and a proper graphical user interface (e.g., a dashboard showing the QoS at each location), it is possible to decide which is the best location to place the new sensor node minimising its impact in the QoS being provided by the network.

\section{Conclusions}

Wireless sensor networks combined with low-power sensor devices, have the potential to integrate the physical world with widely used computing systems. Furthermore, such networks can be used to create ubiquitous and pervasive intelligent systems with the potential to greatly impact our daily lives. Among all the application areas of the wireless sensor network, the healthcare is one of the most promising. In particular, concerning its potential to change not only the today's healthcare services provided to the citizens but also the clinical 
practice. The use of WSNs in healthcare brings several advantages, in particular as regards to the automation of routine processes such as periodic patient monitoring. Automatic patient monitoring systems can be used to complement the episodic measurements made by the healthcare professionals (e.g., body temperature, pulse and respiratory rates, blood pressure or oximetry), bringing out an enhancement of the quality of care, while freeing the nursing staff to provide extra attention to the patients.

Nevertheless, due to the demanding requirements of healthcare services and applications, WSNs have to fulfil high levels of quality of service (QoS) to be fully accepted by the healthcare professionals and patients. As an effort to provide healthcare professionals with a tool to manage such networks, bearing in mind the objective of maximising the QoS provided by WNSs supporting patient monitoring applications, this work proposes a new smart context-aware QoS-based network management method able to manage the admission of new nodes to the network.

The proposed admission control method makes use of a "virtual sensor node" to verify if a new patient (i.e., a new sensor node) can be added to the network and decides which would be the best place to admit the new patient into the network. The use of a "virtual sensor node" avoids the necessity of the new patient to be physically present within the network and enables to assess the on-the-fly QoS provided by the network from a remote location. By using a "virtual sensor node", the proposed admission control method enables the network administrator (n.b., the figure of the network administrator must be seen in a broad sense since it could be either a person or a virtual process) to estimate the presence of a real sensor node within the network from a remote location. This characteristic gives the proposed method an innovative feel, when comparing it with other proposals. Moreover, the possibility to know in advance the best location to place a patient gives the healthcare providers an important tool to optimise their work and organizational processes.

Considering the results obtained from several experiments in realworld environments, it is possible to outcome that the proposed admission control method, based on the use of a "virtual sensor node" to predict the behaviour of the network when introducing the new sensor node, was successfully validated and the conditions necessary to its use were clearly presented.

\section{Acknowledgment}

Work supported by the Portuguese Foundation for Science and Technology, FCT, PhD Grant SFRH/BD/61278/2009. Miranda was supported by Portuguese funds through the CIDMA - Center for Research and Development in Mathematics and Applications, and the Portuguese Foundation for Science and Technology.

\section{References}

Abreu, C., Ricardo, M., Mendes, P.M., 2014a. Energy-aware routing for biomedical wireless sensor networks. J. Netw. Comput. Appl. 40 (0), 270-278.

Abreu, C., Miranda, F., Ricardo, M., Mendes, P.M., 2014b. QoS-based management of biomedical wireless sensor networks for patient monitoring. SpringerPlus 3 (1), 239.

Abreu, Carlos, Mendes, P.M., 2014. In: Miranda, F. (Ed.), Ch. Providing QoS in Wireless Sensor Networks: A System Overview. Nova Science Publishers, Inc, 171-196.

Anjum, Iffat, Alam, Nazia, Razzaque, Md. Abdur, Hassan, Mohammad Mehedi, Alamri, Atif, 2013. Traffic priority and load adaptive MAC protocol for QoS provisioning in body sensor networks. Int. J. Distrib. Sens. Netw. 2013, 9.

Carmen Domingo, Mari, 2012. An overview of the Internet of Things for people with disabilities. J. Netw. Comput. Appl. 35 (2), 584-596, (Simulation and Testbeds).
CC1000, 2005. Single Chip Very Low Power RF Transceiver, Fbr.

CC2420, 2.4 GHz IEEE 802.15.4/ZigBee RF Transceiver, Oct. 2011.

Chen, Jaime, Díaz, Manuel, Llopis, Luis, Rubio, Bartolomé, Troya, José M., 2011. A survey on quality of service support in wireless sensor and actor networks: requirements and challenges in the context of critical infrastructure protection. J. Netw. Comput. Appl. 34 (4), 1225-1239, (Advanced Topics in Cloud Computing).

Chen, Min, Gonzalez, Sergio, Vasilakos, Athanasios, Cao, Huasong, Leung, Victor C., 2011. Body area networks: a survey. Mob. Netw. Appl. 16 (2), 171-193.

Dunkels, A., Gronvall, B., Voigt, T., 2004. "Contiki - a lightweight and flexible operating system for tiny networked sensors," in Local Computer Networks, 2004. 29th Annual IEEE International Conference on, Nov, pp. 455-462.

Farooq, Muhammad Omer, Kunz, Thomas, 2014. BandEst: measurement-based available bandwidth estimation and flow admission control algorithm for ad hoc IEEE 802.15. 4-based wireless multimedia networks. Int. J. Distrib. Sens. Netw. $2014,15$.

Gama, O.S., 2011. A MAC Protocol for Quality of Service Provisioning in Adaptive Biomedical Wireless Sensor Networks (Ph.D. dissertation). University of Minho.

Hadjidj, Abdelkrim, Souil, Marion, Bouabdallah, Abdelmadjid, Challal, Yacine, Owen, Henry, 2013. Wireless sensor networks for rehabilitation applications: challenges and opportunities. J. Netw. Comput. Appl. 36 (1), 1-15.

Ibrahim Orhan, Lindh, Thomas, 2011. Measurement-based performance and admission control in wireless sensor networks. Int. J. Adv. Syst. Meas. 4 (1-2), 32-45.

Ignacio Más, Karlsson, Gunnar, 2007. Probe-based admission control for a differentiated-services internet. Comput. Netw. 51 (13), 3902-3918.

Ignacio Más, Karlsson, Gunnar, 2008. A Model for Endpoint Admission Control Based on Packet Loss. In: Das, Amitabha (Ed.), NETWORKING 2008 Ad Hoc and Sensor Networks, Wireless Networks, Next Generation Internet 4982. Springer Berlin Heidelberg, 506-517. http://dx.doi.org/10.1007/978-3-540-79549-0 44.

Jacob, Anil K., Jacob, Lillykutty, 2015. Energy efficient MAC For QoS traffic in wireless body area network. Int. J. Distrib. Sens. Netw. 2015, 12.

Khan, Zahoor Ali, Sivakumar, Shyamala, Phillips, William, Robertson, Bill, 2014. ZEQoS: a new energy and QoS-aware routing protocol for communication of sensor devices in healthcare system. Int. J. Distrib. Sens. Netw. 2014, 18.

Ko, J., Gao, T., Terzis, A., 2009. Empirical Study of a Medical Sensor Application in an Urban Emergency Department, in Proceedings of the Fourth International Conference on Body Area Networks, ICST, Brussels, Belgium, Belgium, pp. 10:1-10:8

Ko, JeongGil, et al., 2010a. Wireless sensor networks for healthcare. Proc. IEEE 98 (11), 1947-1960.

Ko, JeongGil, 2012. Designing A Low-power Mobile Sensing System For Wireless Healthcare Applications (Ph.D. dissertation). Johns Hopkins University, Baltimore, Maryland.

Ko, JeongGil, Gao, Tia, Rothman, R., Terzis, A., 2010b. Wireless sensing systems in clinical environments: improving the efficiency of the patient monitoring process. Eng. Med. Biol. Mag. IEEE 29 (2), 103-109.

Lai, Chien-Chih, Lee, Ren-Guey, Hsiao, Chun-Chieh, Liu, Hsin-Sheng, Chen, ChunChang, 2009. A H-QoS-demand personalized home physiological monitoring system over a wireless multi-hop relay network for mobile home healthcare applications. J. Netw. Comput. Appl. 32 (6), 1229-1241.

Lindh, T., Orhan, I., 2009. Performance monitoring and control in contention-based wireless sensor networks, in Wireless Communication Systems, 2009. ISWCS 2009. 6th International Symposium on, sept, pp. 507-511.

Mir Mehedi, Ahsan Pritom, et al., 2015. A multiconstrained QoS aware MAC protocol for cluster-based cognitive radio sensor networks. Int. J. Distrib. Sens. Netw., 13.

Nouha Baccour, et al., 2012. Radio link quality estimation in wireless sensor networks: a survey. ACM Trans. Sen. Netw. 8 (4).

Paola, Alessandra De, Re, Giuseppe Lo, Milazzo, Fabrizio, Ortolani, Marco, 2013. QoSaware fault detection in wireless sensor networks. Int. J. Distrib. Sens. Netw. 2013, 12.

Pereira, V., Silva, J.S., Monteiro, E., 2012. A framework for Wireless Sensor Networks performance monitoring, in World of Wireless, Mobile and Multimedia Networks (WoWMoM), 2012 IEEE International Symposium on a, Jun, pp. 1-7.

Polastre, J., Szewczyk, R., Culler, D., 2005. Telos: enabling ultra-low power wireless research. In: Proceedings of the 4th international symposium on Information processing in sensor networks, Piscataway, NJ, USA.

Rappapport, T.S., 2002. Wireless Communications: Principles and Practice 2nd ed. Prentice Hall.

Sun, T., Chen, L., Yang, G., Sanadidi, M.Y., Gerla, M., 2005. SenProbe: Path Capacity Estimation in Wireless Sensor Networks, In: Proceedings of the 3rd International Workshop on Measurement, Modelling, and Performance Analysis of Wireless Sensor Networks.

Yang, Yaling, Kravets, R., 2005. Contention-aware admission control for ad hoc networks. Mob. Comput. IEEE Trans. 4 (4), 363-377.

Zamalloa, M.Z., Krishnamachari, Bhaskar, 2007. An analysis of unreliability and asymmetry in low-power wireless links. ACM Trans. Sen. Netw. 3 (2).

Zuniga, M., Krishnamachari, B., 2004. Analyzing the transitional region in low power wireless links, in Sensor and Ad Hoc Communications and Networks, 2004. IEEE SECON 2004. 2004 First Annual IEEE Communications Society Conference on, Oct, pp. 517-526. 